\title{
Aménagements hydroélectriques fluviaux à buts multiples: résolution du marnage artificiel et conséquences sur les objectifs écologique, énergétique et social
}

\author{
Philippe Heller, Anton Schleiss² \\ Associé - directeur, e-dricch Eau Energie Enwomenent Ingénieurs Conseils Sàt, Chemin du Rionit 54,CH - 1052 Le Mont-sur-Lausame, \\ Suisse, philippe.hellerouedric, ch \\ Directat, Laboratome de Consmictions Hydrauliques, Ecole Polytechnique Fédérale de Latsamne, Station 18, CH-1015 Lausanne, Suisse. \\ unton.schleiss@eptl.ch
}

RESUME. - D'importants travaux sont actuellement entrepris dans le contexte de la gestion des crues et de la renaturation des cours d'eau alpins. Pour bénéficier totalement du potentiel de revitalisation, l'hydrologie doit redevenir aussi naturelle que possible. Dans le cadre de la production d"énergie de pointe des aménagements hydroélectriques à haute chute, la génération du marnage artificiel doit impérativement être réduite. Un aménagement de rétention sur le cours d'eau aval permet de répondre à cette problématique sans renoncer à la production de pointe. L'adjonction d'objectifs supplémentaires pour cet aménagement permet d'augmenter son degré d'acceptabilité. Ces objectifs additionnels sont la production d'énergie, le développement d'activités de loisirs aquatiques, le laminage des crues et l'intégration paysagère. Ainsi devenu un aménagement à buts multiples, sa complexité est accrue et requiert de nouvelles approches. Après une étude qualitative de la compréhension du système et de son environnement, le présent article développe les outils de gestion, de simulation et d'optimisation nécessaires au développement des solutions optimales. Les solutions trouvées pour le cas d'étude sur le Rhône suisse alpin supérieur génèrent plus de $50 \mathrm{GWh} /$ an d'énergie tout en supprimant le marnage artificiel et en offrant un volume de rétention pour le laminage des crues. Le réservoir présente également un potentiel pour plus de $50^{\prime} 000$ visites annuelles pour des loisirs aquatiques.

Mots-clés : aménagement à buts multiples, Rhône suisse alpin supérieur, optimisation des systèmes complexes, centrale hydroélectrique au fil de l'eau.

\section{Multipurpose run-of-river hydroelectric power plants : hydropeaking mitigation and consequences on ecological, energetic and social objectives}

\begin{abstract}
Flood protection and ecological rehabilitation of alpine river need significant river training works. In order to develop their highest ecological potential, such restoration works necessitate a near natural flow regime. Therefore, artificial hydropeaking due to peak energy production should be reduced drastically. A retention basin, directly built on the downstream river could restore a near natural regime without any constraint on the upstream peak energy production. Designed as a multipurpose reservoir project, such a basin could meet a high degree of public acceptation. Beside hydropeaking mitigation, the various purposes of the project are local renewable energy production, aquatic leisure activities, flood routing and landscape integration. Since the assessment of a multi-purpose project induces a high degree of complexity, new approaches are required to maximize the synergies between the different project purposes. This article presents the quantitative simulation and optimisation tools of the complex system formed by such a project and its environment. For the considered case study on the Upper Swiss Rhone River, the obtained configuration of the multipurpose reservoir can produce $50 \mathrm{GWh} / \mathrm{yr}$ and simultaneously restore downstream a near-natural flow regime. The reservoir also allows attracting more than $50^{\prime} 000$ visits per year for leisure activities.
\end{abstract}

Key-words : complex system optimisation, multi-purpose scheme, run-off-river power plant, Swiss Rhone River.

\section{INTRODUCTION}

A côté de la sensibilité croissante aux problèmes environnementaux, les changements climatiques pressentis montrent des situations météorologiques extrêmes aggravées. Par ailleurs, les populations installées à proximité des rivières réduisent fortement l'espace de ces demières. Le risque global a ainsi fortement augmenté et les conséquences sont devenues d'autant plus inacceptables. Cet état de fait nécessite, pour des raisons sécuritaires principalement, d'importants travaux sur les cours d'eaux
Dans les pays développés où le potentiel hydroélectrique est déjà largement développé, la réalisation de nouveaux aménagements est devenue difficile. Des contraintes écologiques, sociales ou politiques font souvent obstacles a des projets intéressants mais analysés du seul point de vue technique ou économique. Pour répondre à ce problème, de nombreuses méthodes participatives ont été développées (Leach \& al., 2001). Elles incluent, dès les premières phases du projet, l'ensemble des partenaires potentiels et ont pour but d'aboutir à un compromis raisonnable. Pour repondre a l'ensemble des aftentes, elles favorisent les projess a buts multiples (Schleiss, 2006). 
Selon Flug \& al. (2000) et Cai \& al. (2004), les buts possibles pour un barrage fluvial se répartissent dans trois catégories: hydraulique, écologique et socio-économique. La production énergétique, la protection contre les crues, l'irrigation et la navigation forment la première catégorie. Lécologie des rivières, la réduction du marnage artificiel et la création de biotopes forment la seconde catégorie. La pèche. les zones de récréation et l'intégration paysagère constituent la dernière catégorie.

Un projet à buts multiples génère un système complexe avec des effets rétroactifs. Son étude doit considérer les perspectives de tous les acteurs. Les variables du projet avec leurs rétroactions ainsi que l'environnement dans lequel le projet s'inscrit doivent être modélisés avec consistance.

La littérature suggère d'analyser les systèmes complexes successivement par des modèles qualitatif et quantitatif (Gomez and Probst, 1995, Coyle, 2000). Les systèmes dynamiques sont définis comme "une méthode d'analyse des systemes complexes faite par une analyse causale, suivie d'une transcription dynamique, complétée par un modèle et terminée par une simulation informatique " (EuroDicauTom, 1982). La considération de chacun des aspects proposés du projet à buts multiples requiert ainsi une analyse qualitative. Dans un second temps, l'analyse quantitative doit spécifier les aspects purement numériques. Le but de cette méthodologie appliquée à des projets hydrauliques est de développer des synergies au sein de ces ouvrages de sorte à augmenter leur degré d'acceptabilité.

Les aspects qualitatifs de la méthode proposée par la dynamique des systèmes sont appliqués aux aménagements hydroélectriques fluviaux à buts multiples et présentés dans Heller et al. (2007). Cette étude qualitative définit les principaux objectifs de l'aménagement. Ce sont le laminage du marnage, la production d'énergie, la rétention des crues, le developpement d'activités de loisirs aquatiques et la stabilité du niveau d'eau dans le réservoir. Cet article présente

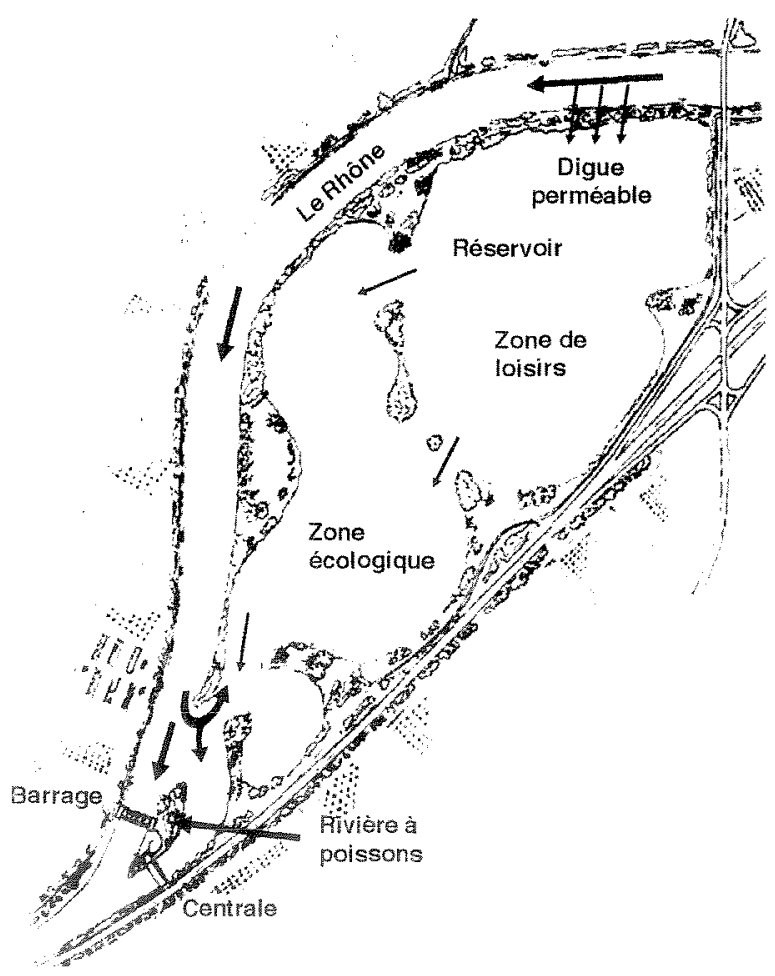

rigure 1: Aménagenent à buts mulliples sw to Rhone suisse alpin superieur. spécifiquement la gestion ordinaire d'un tel aménagement et ses conséquences sur les objectifs écologique, énergétique et social. Le cas d'étude est situé sur le Rhòne suisse alpin supérieur (canton du Valais) et illustré par la Figure 1 (Bollaert \& al., 2000).

\section{ALGORITHME DE RESOLUTION}

L'étude qualitative de l'aménagement à buts multiples (Heller, 2007) met en évidence la variable de gestion comme variable pivot du système complexe. Cette demière constitue le lien physique entre le modèle conceptuel de décision et le modèle physique de fonctionnement de l'aménagement. En effet, d'une part, les valeurs des variables de dimensionnement de l'aménagement dépendent de la gestion souhaitée de ce dernier et résument ainsi toutes les réflexions menées sur le projet. D'autre part, la gestion constitue l'ultime variable de décision du système et conditionne tout le fonctionnement physique de l'aménagement. Cette variable intervient donc en dernière position dans l'ordre de la réalisation mais en première position dans l'ordre de la réflexion. Il convient ainsi de définir dans un premier temps la gestion souhaitée du réservoir et d'en déduire les conséquences sur les autres objectifs du projet (écologie, énergie, utilité sociale). Cette réflexion est synthétisée par l'algorithme présenté à la Figure 2.

Cet algorithme est divisé en trois étapes principales. La première étape concerne les variables externes et résument toutes les influences externes de l'environnement sur le projet étudié. Elles caractérisent ainsi par exemple un site potentiel le long du cours d'eau par les débits qui s'y écoulent ou les températures de l'air et de l'eau qui y règnent. La seconde étape détermine la gestion du réservoir à buts multiples. Elle utilise les variables externes comme données et, avec ses propres variables, définit le débit aval de l'aménagement. La gestion distingue les situations hydrologiques de crue de la situation ordinaire. Seule la situation ordinaire est présentée dans cet article. Enfin, la troisième étape intègre l'ensemble des conséquences de la gestion sur l'aménagement et le cours d'eau aval. Ces modèles de simulation reprennent les principaux objectifs de l'aménagement, à savoir les aspects écologique, énergétique et social, tous dépendant directement des aspects hydrauliques.

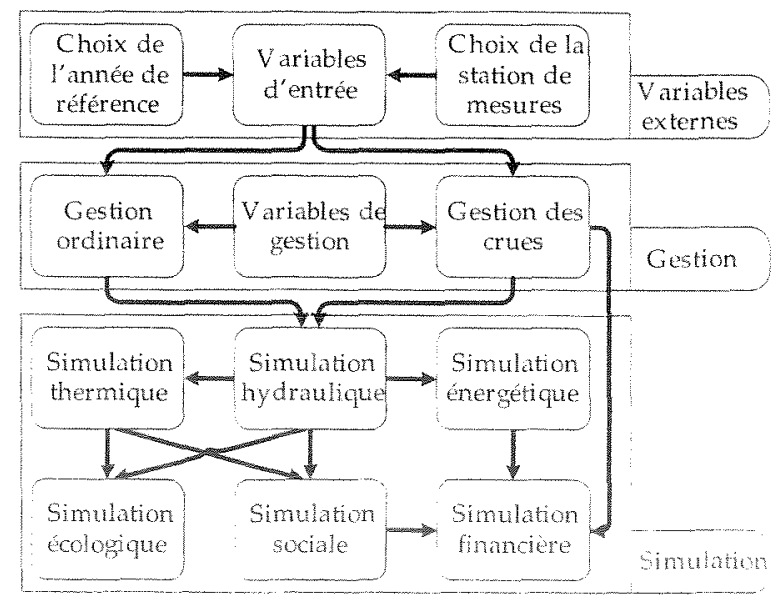

Figure 2: Algorithe de gesion d'un amenagement a buts multiples a comme un systeme conplexe. 


\section{MODELE DE GESTION DU RESERVOIR}

L'optimisation présentée porte uniquement sur la gestion hydraulique ordinaire de laménagement (situation hors crue). Le modèle de gestion vise simultanément quatre objectifs. Il s'agit tout d'abord de réduire, voire de supprimer, le marnage artificiel produit dans le Rhône par les restitutions des centrales à accumulation. Ensuite, il tend à minimiser les oscillations du niveau d'eau dans le réservoir, à maximiser la production d'énergie ainsi qu'à stabiliser le niveau dans le réservoir spécifiquement pendant la période estivale à des fins de loisirs aquatique. Le premier objectif, qui constitue également l'objectif principal du projet, est introduit dans le modèle mathématique par deux contraintes additionnelles sur les oscillations du niveau dans la rivière aval, à savoir une amplitude hebdomadaire maximale $\left(A_{\mathrm{av}}\right)$ et un gradient maximal $\left(\Delta h_{a v}\right)$. La fonction objective du modèle mathématique vise la maximisation du taux moyen de remplissage du réservoir $(T)$ calculé sur une base horaire. En maximisant la chute disponible, cette fonction maximise implicitement la production d'énergie. Compte-tenu de la dérivée strictement croissante du volume du réservoir par rapport à son taux de remplissage, cette même fonction objective minimise également les oscillations dans le réservoir ce qui conduit à une meilleure stabilisation du niveau. Les équations 1 à 5 définissent mathématiquement le modèle de gestion proposé.

Maximise :

$$
T=\sum_{i=1}^{n} \frac{H_{r e s}(t)}{H_{r e s, \max }}
$$

sous contraintes de :

$$
\begin{aligned}
& h_{a v}(t) \in\left[h_{m w y}-\frac{A_{a w}}{2} ; h_{m o y}+\frac{A_{t a v}}{2}\right], \forall t \\
& \left|h_{a t}(t)-h_{t a}(t-1)\right| \leq \Delta h_{a,}, \forall t \\
& H_{r e s}(t) \in\left[H_{r e s, \text { min }} ; H_{r e s, \text { max }}\right], \forall t \\
& H_{i r, \min } \geq h_{u v}(t), \forall t
\end{aligned}
$$

où $H_{\text {rebs }}$ représente le volume dans le réservoir au temps $t$, $H_{\mathrm{res}, \max }$ et $H_{\mathrm{res}, \min }$ les volumes maximal et minimal du réser- voir, $h_{\text {moy }}$ le niveau moyen visé dans le cours d'eau aval pour la période comprise entre 1 et $n$ et $h_{\text {as }}$ le niveau dans le cours d'eau aval au temps $t$. Le modèle n'impose pas de limite sur le débit aval. 11 suppose que la capacité du système est supérieure aux débits proposés par le modèle. Pour respecter l'écoulement du réservoir vers la rivière aval, le modèle impose que $H_{\mathrm{res}}$ soit en tout temps supérieur à $h_{3 \mathrm{~s}}$. Ainsi défini, le modèle possède trois variables internes, soit le gradient aval $\Delta h_{x x}$, l'amplitude aval $A_{i \mathrm{x}}$ et l'horizon de planification $n$. Le modèle mathématique est résolu par le logiciel AMPL (Fourer et al., 2003) dans sa version gratuite limitée à 300 variables et contraintes. L'horizon de planification est ainsi limité à 96 heures, réactualisé toutes les 48 heures. Sur cet horizon, le modèle admet une prévision parfaite des débits. Les contraintes écologiques sur le gradient et l'amplitude possèdent chacune une valeur hivernale et une valeur estivale. La saisonnalité est définie en fonction du débit hebdomadaire moyen.

\section{MODELE DE SIMULATION HYDRAULIQUE}

Le modèle hydraulique simule les conséquences de la gestion sur les niveaux d'eau dans la rivière aval et dans le réservoir. Il applique l'équation de bilan au réservoir (pas de temps horaire). La Figure 3 illustre la gestion optimale proposée pour une semaine hivernale type. Les contraintes sur les gradients hivernal et estival valent 8 et $12 \mathrm{~cm} / \mathrm{h}$ (Halleraker et al., 2003) et respectivement 30 et $40 \mathrm{~cm}$ pour les amplitudes (Meile et al., 2005).

Pour la période hivernale, les conséquences de la gestion optimale sont une oscillation journalière dans le réservoir de l'ordre de $60 \mathrm{~cm}$ et une oscillation hebdomadaire de l'ordre de $2.50 \mathrm{~m}$. Cet abaissement du niveau du réservoir permet de soutenir le débit faible pendant les jours fériés. En contre partie, le niveau de la rivière aval est largement tranquillisé. Ses variations journalières de $100 \mathrm{~m}^{3} / \mathrm{s}$ sont réduites à une seule variation hebdomadaire de $35 \mathrm{~m}^{3} / \mathrm{s}$. Pendant la période estivale, le réservoir est très peu sollicité et son niveau d'eau varie très peu. Il assure seulement le respect de la contrainte sur le gradient. Cette gestion est favorable pour les activités de loisirs.

L'agrégation annuelle du gain hydrologique est effectuée à l'aide d'un indicateur global (Meile et al., 2005). Il repré-

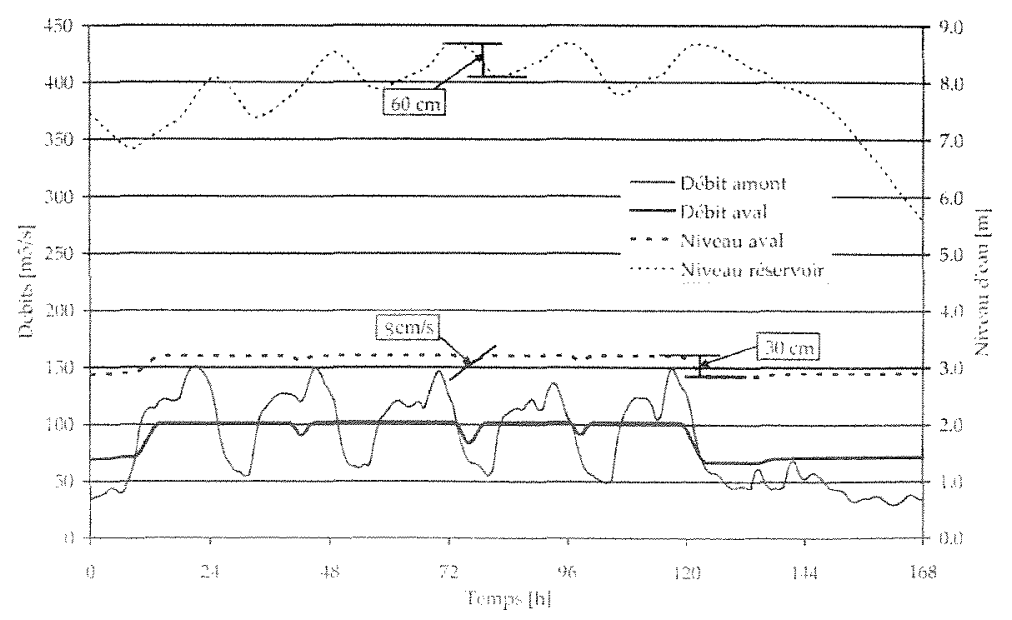

Figure 3: Débits et niveatx pour une semaine hivernale type. 
sente la probabilité entre deux heures successives, calculée sur un cycle annuel, d'obtenir une variation du niveau d'eau égale ou inférieure à une valeur donnée. La Figure 4 illustre cet indicateur pour trois séries hydrologiques distinctes. La courbe au somment représente la référence historique du cours d'eau dans son état quasi-naturel (débit de l'année 1907). La courbe en gras représente l'état actuel. La stabilité du niveau, historiquement présente pendant $70 \%$ du temps, est réduite à $15 \%$. La courbe grasse en trait tillé indique la rivière aval avec une gestion optimale. La majeure partie des variations est absorbée par le réservoir. La tranquillité entre deux heures successives rejoint la valeur historique. La dernière courbe illustre les oscillations du réservoir. Malgré la concentration du marnage artificiel, ce dernier possède une tranquillité supérieure à l'état actuel. Ainsi, la courbe de remous amont présente des caractéristiques hydrologiques également améliorées.

\section{MODELE DE SIMULATION ECOLOGIQUE}

A cause de la différence d'échelle, le modèle d'écologie aquatique traite distinctement le réservoir et la rivière à poissons de la rivière aval. Cette dernière constitue par la gestion optimale le principal gain écologique escompté. Elle fait ainsi l'objet d'un modèle de simulation propre. Le réser- voir et la rivière à poissons sont traités uniquement par des aspects constructifs bases sur l'expertise.

\section{V.1. Réservoir et rivière à poissons}

Les oscillations du niveau d'eau dans le réservoir posent, en plus de la sécurité structurale des berges, un problème d'intégration architecturale et de développement écologique de l'écotone. Pour permettre un contact permanent de l'écotone à l'eau, la majeure partie des berges possède un ourlet écologique à l'intérieur du réservoir (Pellaud, 2007). Ce dernier conserve un niveau d'eau permanent. La Figure 5 illustre un tel ourlet.

La rivière à poisson assure le continuum écologique interrompu par le barrage et la centrale de production hydroélectrique. Elle constitue un véritable système écologique en permettant le transit longitudinal de l'ensemble de la faune aquatique. Ses principales caractéristiques sont un débit important (environ 10 fois supérieur à celui d'une passe technique, soit $5 \mathrm{~m} / \mathrm{s})$, une pente faible $(1.1 \%)$ et une alternance de rapides et de mouilles.

\section{V.2. Rivière aval}

La rivière aval fait l'objet d'un modèle de simulation d'écologie aquatique (Pellaud, 2007) basé sur le principe

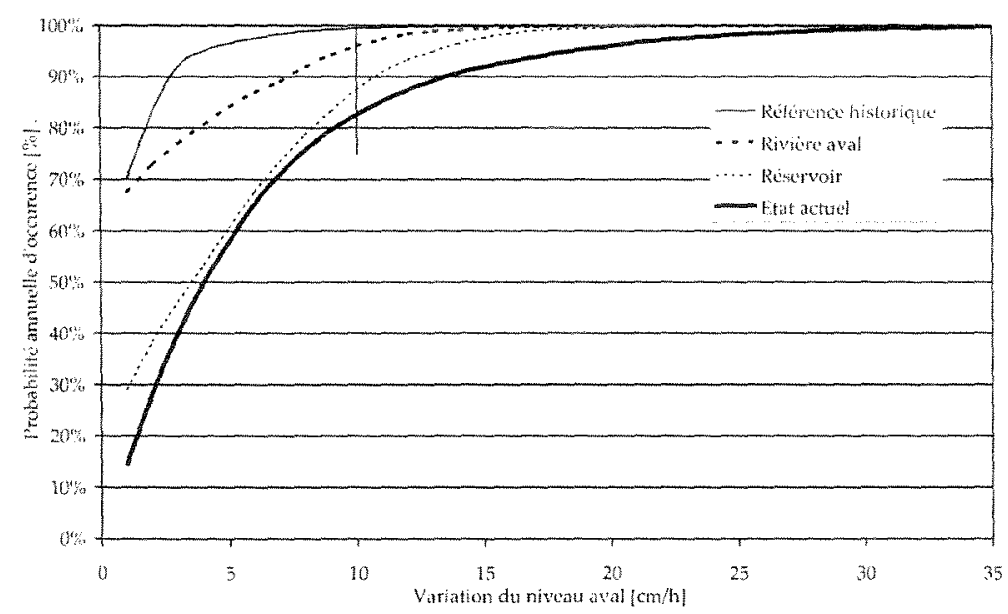

Figure 4: Occurrence d'une variation de niveau d'eau entre deux heures successives.

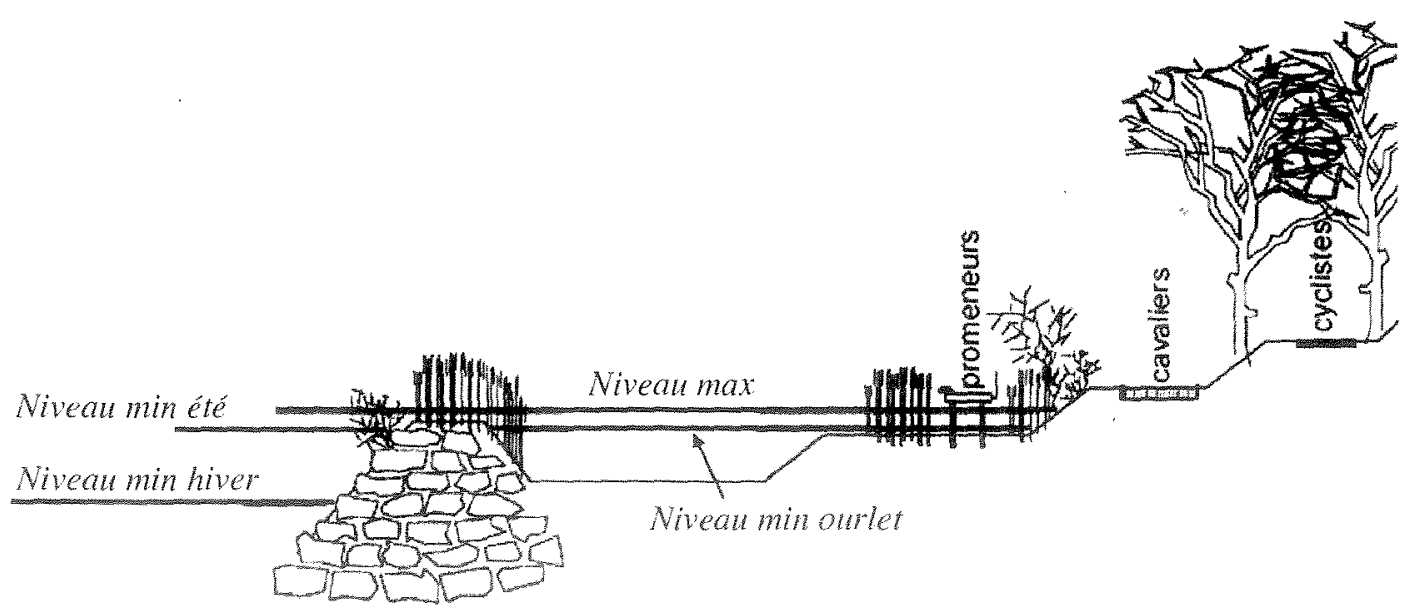

Figure 5: Oullet écologique sur les berges du résenoir : gain écologique et paysager: 
admis que la qualité écologique peut être quantifiée par la diversité des communautés aquatiques présentes (Poff, 1997). Ce modèle fonctionnel distingue deux modules différents pour les poissons et les invertébrés. Tous deux intègrent des données statistiques basées sur des relevés taxononiques ainsi que l'effet de la suppression du marnage artificiel. Les différentes valeurs obtenues sont agrégées pour aboutir à une seule note représentative de la qualité écologique du cours d'eau.

Pour les poissons, le modèle divise l'habitat en quatre guildes, soit les berges, les mouilles, les radiers et le cours plein (Lamouroux et al., 2002). Il calcule, pour chaque guilde, une valeur d'habitat sur la base d'un relevé statistique et l'ajuste ensuite par un modèle de logique floue (Valentin, 1995) en fonction de l'hydrologie et des modifications thermiques. La valeur d'habitat peut être finalement multipliée par la surface du cours d'eau pour obtenir une surface utile à chaque guilde.

Pour les invertébrés, le modele considère trois taxons principaux par un indicateur EPT qualifiant la richesse d'un milieu en insectes, à savoir les Ephéméroptères $(E)$, les Plécoptères $(\mathrm{P})$ et les Trichoptères $(\mathrm{T})$. Des fonctions radiales (RBF) (Lek et al., 1999, Obach et al., 2001, Zhang et al., 2005) sont utilisées pour calculer un indice standard de richesse (Preliminary Standardized Richness Index). Cet indice prédit la richesse du cours d'eau soumis aux conditions hydrologiques similaires à celles des mesures taxonomiques. Un coefficient modifie alors cette richesse en fonction de la suppression du marnage sur la base d'un modèle de logique floue (Silvert, 2000, Adriaenssens et al., 2004).

\section{MODELE DE SIMULATION ENERGETIQUE}

Le modèle de simulation énergétique calcule pour chaque heure, en fonction des niveaux amont et aval et du débit disponible, la puissance produite. Il résume la chaîne de transformation énergétique à un seul rendement global. $\mathrm{Ce}$ coefficient est calculé sur la base d'une optimisation des principaux paramètres géométriques des machines hydrauliques (diamètre des roues, vitesse de rotation et profondeur d'implantation). Cette optimisation est effectuée dans le cadre d'une forte variabilité du débit et de la charge. Indépendamment de la hauteur du barrage, du débit équipé de la centrale ou de la surface du réservoir (influence réduite de ce dernier facteur), il est toujours possible de trouver une combinaison raisonnable des principaux paramètres, exprimée par des coûts d'installation, avec un rendement annuel global de $85 \%$ au minimum.

La gestion énergétique du réservoir qui maximise la production constitue la référence absolue. Cette gestion consiste à garder le niveau d'eau amont constamment à sa cote maximale d'exploitation. Elle est formalisée par l'équation 6 .

$$
Q_{o u t}=Q_{n}
$$

où $Q_{3}$ est le débit qui entre dans l'aménagement et $Q_{\text {ins }}$ le débit aval. Cest la gestion habituelle des aménagements hydroelectriques au fil de l'eau. Par mapport à cette gestion, la gestion optimisée génère une perte énergétique comprise entre 5 et $10 \%$ selon les valeurs des variables internes du modele de gestion.

\section{MODELE DE FREQUENTATION DU SITE}

Le modèle de fréquentation du site calcule le nombre annuel de visites liées à des activités aquatiques dans le réservoir. Il est basé sur un site existant similaire (anciennes gravières transformées en étangs naturels). L'évolution mesurée du chiffre d'affaire du restaurant du site de référence permet de reproduire l'évolution de la fréquentation globale de ce dernier. Sur la base d'une étude statistique régionale portant sur les activités aquatiques, la fréquentation est répartie en fonction des activités possibles. Cette même étude permet également de ventiler la fréquentation en fonction de la distance parcourue pour se rendre sur le site. La transposition sur le site projeté tient ainsi compte de la répartition de la population autour du site et des activités développées. Des fonctions de préférence basées sur la température de l'air et de l'eau ainsi que sur les oscillations du niveau dans le réservoir minorent la fréquentation journalière. La fréquentation est essentiellement condensée sur les trois principaux mois estivaux.

\section{APPLICATION AU CAS D'ETUDE}

A cause de ses nombreux réservoirs alpins hydroénergétiques, le régime hydraulique du Rhône suisse alpin supérieur subit une influence artificielle journalière et saisonnière (marnage journalier et report saisonnier). Les nombreux glaciers du bassin versant provoquent dans le Rhône une eau fortement chargée en sédiments fins. A la suite de deux corrections effectuées à la fin du XIX ème et au milieu du XX $\mathrm{XX}^{\text {ìme }}$ siècle, le cours d'eau est fortement canalisé sur l'intégralité de sa longueur (environ $160 \mathrm{~km}$ ) et maintenu par des berges pentues. La succession de trois crues destructrices (1987, 1993 et 2000) d'une période de retour estimée à 100 ans pour chacune a montré la nécessité d'une troisième correction. Parallèlement à l'augmentation de la capacité, cette correction intègre des aspects de renaturation et de développement socio-économique. Les travaux de planification de ce projet sont actuellement en cours (Troisième Correction du Rhône, 2006).

Dans ce contexte, un aménagement à buts multiples est envisagé $(50 \mathrm{~km}$ à l'amont de l'embouchure dans le lac Léman). Pour rappel, ses principaux objectifs présentés dans cet article sont le laminage du marnage, la production d'énergie, le développement d'activités de loisirs aquatiques et la stabilité du niveau d'eau dans le réservoir. Pour les simulations, l'année 1993 est utilisée. Elle correspond le mieux à une année moyenne du point de vue hydrologique et thermique. L'aménagement projeté possède une surface de $1 \mathrm{~km}^{2}$ et une hauteur de $8.70 \mathrm{~m}$ (non-compris la revanche de $1 \mathrm{~m}$ pour les aspects sécuritaires). Le débit de la rivière à poissons est posé a $5 \mathrm{~m}^{3} / \mathrm{s}$. Le débit équipé de la centrale hydroélectrique est admis à $200 \mathrm{~m}^{3} / \mathrm{s}$ ce qui équivaut au

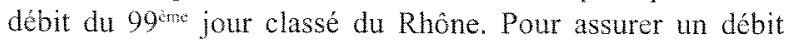
permanent d'environ $5 \mathrm{~m}^{3} / \mathrm{s}$ à travers le réservoir, ce dernier possède, à l'amont, une digue perméable directement en contact avec le Rhône. Ce débit est nécessaire tant pour les aspects de sédimentation que d'ajustement de la température dans le réservoir. Les aspects de sédimentation du réservoir et la forme spécifique de l'entrée aval de ce dernier ont fait l'objet d'une étude particulière pour éviter les problèmes d'ensablement du réservoir (Bollaert \& al., 2000). Au besoin, des purges du barrage en riviere peuvent soutenir le transport solide grossier. 


\section{VIII.I. Etat actuel}

En l'état actuel, le site de Riddes possède un potentiel énergétique brut inexploité d'environ $60 \mathrm{GWh}$ par année. L'absence d'aménagement touristique et les berges pentues du cours d'eau ne permettent aucun contact à l'eau pour des loisirs aquatiques. Enfin, l'intégralité du marnage artificiel demeure sur le linéaire de la rivière. Sur les $2.5 \mathrm{~km}^{2}$ de la surface aval du cours d'eau, seul $10 \%$ sont utiles pour les mouilles, $2 \%$ pour les berges et moins de $1 \%$ pour les radier. La richesse EPT prédite des macro-invertébrés en insectes, à savoir les Ephéméroptères (E), les Plécoptères $(P)$ et les Trichoptères $(T)$, donne une valeur moyenne de 4.75 taxon par type (Pellaud, 2007). Le Tableau 1 résume les principales caractéristiques de l'état existant du site de Riddes et de la rivière aval.

\section{VIII.2. Scénario avec un aménagement à buts multiples}

La réalisation d'un aménagement à buts multiple permet la génération d'environ $41 \mathrm{GWh}$ par année. Cette production atteint les $70 \%$ du potentiel brut. Compte-tenu de la production prévue en ruban et de l'extrapolation économique sur le très long terme (50 ans), cette dernière est valorisée avec un prix moyen de $9.5 \mathrm{ct} / \mathrm{kWh}$ (prix calculé avec un contrat moyen de base sur la bourse électrique www.eex. com pour 2006). Elle génère ainsi un revenu annuel moyen de 3.95 Mio CHF. Parallèlement, selon la Figure 4, le marnage est largement réduit. Limité au marnage fort (variation horaire supérieur à $10 \mathrm{~cm} / \mathrm{h}$ ) et en admettant que la surface entre les différentes courbes représente linéairement le marnage destructeur, la gestion proposée supprime $91 \%$ de ce demier pour la rivière aval et $64 \%$ dans la courbe de remous amont. Cette tranquillisation se traduit, dans le modèle écologique, par un léger gain de surface utile pour les poissons $(+5 \%)$ et surtout une très forte amélioration de l'indice de

\section{Tablean 1: Etat actuel du Rhône selon les indicateurs} développés.

\begin{tabular}{|c|c|c|}
\hline Whatution d'énergie & -- & GWh/an \\
\hline Pumentation aquatique du site & - & Visites/an \\
\hline Amancur de berges accessibles & -. & $\mathrm{km}$ \\
\hline builles & $268 \cdot 750$ & $\mathrm{~m}^{2}$ \\
\hline Berges & $45^{\prime} 500$ & $\mathrm{~m}^{2}$ \\
\hline Radier & $12 \times 250$ & $\mathrm{~m}^{2}$ \\
\hline Cours plein & $813 \cdot 750$ & $m^{2}$ \\
\hline Richesse EPT & 4.75 & -- \\
\hline
\end{tabular}

Tableau 2 : Etat du Rhone avec un aménagement à buts multiples.

\begin{tabular}{lrc}
\hline Production d'énergie & 41.5 & GWh/an \\
Frequentation aquatique du site & $54^{\prime} 100$ & Visites/an \\
Eongueur de berges accessibles & $\sim 1$ & $\mathrm{~km}$ \\
Moulles & 265.000 & $\mathrm{~m}^{2}$ \\
Berges & 44000 & $\mathrm{~m}^{2}$ \\
Radier & 10.500 & $\mathrm{~m}^{2}$ \\
Cours plein & $880 \cdot 500$ & $\mathrm{~m}^{2}$ \\
Richesse EPT & 14.80 & $\ldots$ \\
\hline
\end{tabular}

richesse des macro-invertébrés $(+211 \%$ ) (Pellaud, 2007). Le modèle de fréquentation estime le nombre annuel de visite à $54^{\circ} 000$ unités. Le Tableau 2 résume les principales caractéristiques du site de Riddes et de la rivière aval avec un aménagement à buts multiples.

La correction du Rhône suisse alpin supérieur vise un élargissement du lit principal de la rivière d'environ $50 \%$ ansi que la suppression du lit majeur. Cet élargissement permet une augmentation de la capacité de la rivière pour les débits de crues, une amélioration écologique supposée par des berges moins pentues ainsi qu'un contact amélioré à l'eau pour des activités aquatiques.

\section{VIII.3. Scénario avec un aménagement à buts multiples et un cours d'eau corrigé}

Les conséquences de l'élargissement ont un effet direct sur la production d'énergie par l'abaissement du niveau aval moyen. Avec une augmentation moyenne de la chute de $93 \mathrm{~cm}$, la production est augmenté de $23 \%$. Le nombre de visite pour des loisirs aquatiques sur le site est très peu modifié. L'abaissement du niveau moyen dans la rivière aval nécessite, pour les mêmes contraintes imposées sur les variations du niveau, des contraintes légèrement plus élevées sur les débits. Le réservoir est ainsi légèrement plus sollicité, notamment en été, ce qui conduit à une petite baisse de la fréquentation. Le linéaire du Rhône profite également d'un contact à l'eau possible. Cette amélioration n'est pas quantifiée avec la fréquentation du site. D'un point de vue écologique, l'indice de richesse prédite des macro-invertébrés est augmenté de $380 \%$ par rapport à l'état existant et la surface utile pour les quatre guildes de poissons de $109 \%$ (Pellaud, 2007). Compte tenu d'une augmentation de la surface totale de $50 \%$, le gain de surface utilisable pour ces dernières lié à l'amélioration du régime hydrologique et de la structure géomorphologique est de $59 \%$. Le tableau 3 résume la combinaison d'un aménagement à buts multiples avec un cours d'eau élargi.

\section{OPTIMISATION DES VARIABLES DE GESTION}

La superposition d'un optimiseur sur l'outil de gestion permet de modifier les valeurs des quatre variables internes de gestion (amplitude et gradient admissibles dans la rivière aval, conditions estivales et hivernales) et d'étudier les conséquences des différentes gestions optimales sur les indicateurs développés. L'optimiseur multi-objectif utilisé (Leyland, 2002) est basé sur le principe des algo-

Tableat 3: Etat du Rhöne avec un aménagement à buts multiples et un cours d'eau élargi.

\begin{tabular}{lrc}
\hline Production d'énergie & 51.1 & GWh/an \\
Fréquentation aquatique du site & $53^{\prime} 500$ & Visites/an \\
Longueur de berges accessibles & -40 & $\mathrm{~km}$ \\
Mouilles & $552^{\prime} 000$ & $\mathrm{~m}^{2}$ \\
Berges & $363^{\prime} 000$ & $\mathrm{~m}^{2}$ \\
Radier & $136^{\prime}, 900$ & $\mathrm{~m}^{2}$ \\
Cours plein & $1334^{\prime} 000$ & $\mathrm{~m}^{2}$ \\
Richesse EPT & 22.70 & $\cdots$ \\
\hline
\end{tabular}




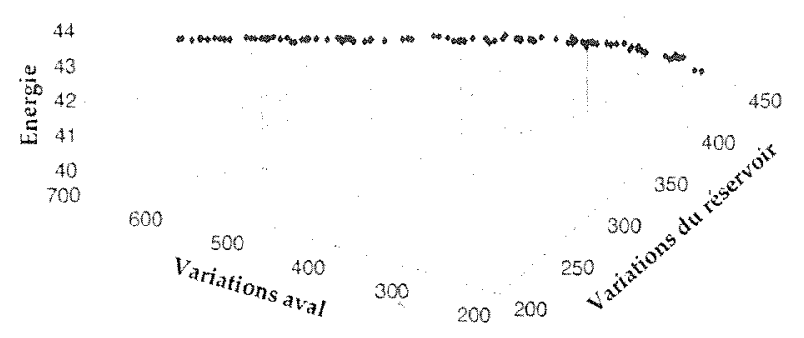

Figure 6: Optimisation selon Pareto avec la production d'énergie (GWh/an), les vartations annuelles du plan d'eau aval $\left(\mathrm{m}^{3} / \mathrm{s} / \mathrm{min} / \mathrm{an}\right)$ et les variations annuelles du plan d'eau dans le réservoir (mian). L'axe vertical commence à 40 GWh/an.

rithmes génétiques et produit un ensemble de solutions optimales au sens de Pareto. La Figure 6 donne pour la production d'énergie, le marnage aval et les oscillations dans le réservoir, l'ensemble des solutions optimales. Elle illustre, avec la projection des solutions obtenues dans le plan « variations aval - variations du réservoir», la dépendance physique, exprimée par l'équation de la continuité, qui relie ces deux axes. La concentration du marnage dans le réservoir stabilise le niveau aval. Inversement, la tranquillité du niveau dans le réservoir est respectée en laissant le marnage dans la rivière aval. Toutefois, le marnage aval est susceptible d'être réduit par un facteur 3 (de 600 à $200 \mathrm{~m}^{3} / \mathrm{s} / \mathrm{min} / \mathrm{an}$ ) pour une augmentation des oscillations dans le réservoir d'un facteur 2 (de 225 à $450 \mathrm{~m} /$ an). L'unité du marnage (rivière ou réservoir) représente la somme annuel des variations du niveau dans le réservoir $(\mathrm{m} / \mathrm{an})$ ou dans la rivière aval (variation du débit entre deux heures successives). La troisième dimension indique la production d'énergie. Cette dernière oscille dans une proportion beaucoup plus faible (entre 40 et $44 \mathrm{GWh} / \mathrm{an}$ ). La stabilité du réservoir conduit à la majoration de la production. Ainsi, pour une gestion aval donnée, l'augmentation de la surface du réservoir conduit à une augmentation de la production. La production calculée ici ne tient pas compte d'un élargissement de la rivière aval et d'un abaissement potentiel du niveau d'eau aval.

Ce résultat constitue une synthèse, pour un aménagement donné, des solutions optimales, physiquement possibles, et de la répartition du marnage amont entre la rivière aval et le réservoir. Les conséquences énergétiques sont directement corrélées.

\section{CONCLUSIONS}

Dans le cadre de la gestion des crues, des travaux importants de corrections sur les cours d'eau sont nécessaires. La législation en vigueur oblige, pour tous travaux de ce type, d'intégrer la dimension écologique. Pratiquement, ceci se traduit principalement par un élargissement du cours d'eau, un abaissement de son niveau moyen et un aplatissement de la pente de ses berges. Dans ce contexte, une hydrologie artificielle fortement variable n'est pas compatible et détruirait tout le potentiel de revitalisation. II est ainsi nécessaire de réduire cette variabilité et de reproduire un hydrogramme plus naturel. Cet objectif peut être facilement atteint avec un réservoir à buts multiples géré activement sur le cours d'eau qui minimise l'espace nécessaire et conserve la production énergétique des aménagements amont.

Avec la nécessité d'un réservoir géré activement et donc la présence d'une chute à l'aval de l'aménagement, la production locale d'énergie s'inscrit dans la logique du projet. Le modèle de gestion optimisée basée sur la prévision des débits montre clairement le potentiel de réunion de deux objectifs apparemment antagonistes. Moyennent une légère perte énergétique (inférieure à $10 \%$ du maximum théorique de production), une réduction du marnage à plus de $90 \%$ peut être atteinte. Les principaux gagnants de cette nouvelle situation sont les macro-invertébrés avec une croissance calculée de près de $400 \%$ (Pellaud, 2007). Cette très forte augmentation est largement issue de l'état actuel très pauvre de la rivière. Elle est liée tant à l'augmentation de la surface du cours d'eau qu'à l'augmentation de la surface utile par tranquillisation du débit.

Les exigences faibles liées au développement de loisirs aquatiques dans le réservoir permettent d'introduire facilement cet objectif dans le projet. La stabilité souhaitée du niveau d'eau dans le réservoir pendant la période estivale recoupe les objectifs de production énergétique et d'intégration paysagère.

La considération des processus physiques liés à un réservoir permet de développer un algorithme simple de simulation de l'aménagement et d'en chiffrer, par des indicateurs corrélés, les conséquences attendues. Des solutions de compromis sont ainsi faciles à développer. L'adjonction d'un outil d'optimisation débouche sur les combinaisons optimales possibles entre les différents objectifs. La compréhension du système et de son fonctionnement lié à la puissance du calcul informatique permettent ainsi de développer les meilleures solutions multi-objectives pour un aménagement bydraulique complexe.

\section{REMERCIEMENTS}

Le projet SYNERGIE est financé par la Commission Fédérale pour la Technologie et l'Imnovation (Switzerland, projet CTI n 6794.1 FHS-IW) ainsi que par l'Office Fédéral de l'Environnement (Switzerland, projet Rhône-Thur, module IV, sous-module 4), l'Etat du Valais et les Forces Motrices Valaisanne. Le laboratoire de gestion des écosystèmes (EPFL, Switzerland), le bureau d'ingénieurs STUCKY et le groupe ANDRITZ-VATECH ont apporté leur soutien dans les domaines écologiques et techniques respectivement.

\section{REFERENCES}

11] Adriagnssens V, Debaets B. And Al. (2004) - Fuzzy mulebased models for decision support in ecosystem management. The Science of the Total Eniroment. $3191-12$

2] Bollaert E., IRNGer P. ANo SChuESS A. (2000) Management of sedimentation in a multipurpose reservoir in a rum-of-river powemplant project on an alpine river. Proc. of conf. Hyiro 2000, Ben, Switerland. $183+192$

(3) CXI X., LASDON L. AND MCHELSEN A.M. (2004) - Group Decision Making in Water Resources Plaming Using Multiple Objective Analysis. I. Whe Resont Phg. Mgm, 130(1) 4-14

[4] CoYLE G. (2000) - Qualitutive and quantiative modelling in system dymmics: some research questions. Sycton Dymanics Revicm. $16225-244$ 
[5] Eurodicautom, A. - Glossary of Space Temm, Esa 1982, Http :/Europa.Eu.Int/Eurodicautom/Controller

[6] Flug M., Sertz H. ANd ScotT J.F. (2000) - Multicriteria Decision Analysis Applied to Glen Canyon Dam. J. Water Resou: Phtg. Mgmt. 126(5) 270-276

[7] Fourer R., Gay D. M. And Kernighan B. W. (2003) AMPL: A Modeling Language for Mathenatical Programming, Diwbury Press, Brooks/Cole Publishing Company, USA

18] Gomez P. Ano Probst G. (1995) - Die Praxis des ganzheitiChen Prohlemlösens, Paul Haupt Verlag, Bem, Switzerland

[9] Halleraker J.H., Saltveit S.J., Harby A., Arnekleiv J.V. FJELDSTA H.-P. AND KohlER B. (2003) - Factors influencing stranding of wild juvenile brown trout (salmo truita) during rapid and frequent flow decreases in an artificial stream. River Res. Applic. 19 589-603

10] HeLler P. (2007) - Méthodologie pour la conception et la gestion des aménagements hydrauliques à buts multiples par une analyse systémique, thèse de doctorat $17^{\circ} 3781$. Communication 30, Laboratoire de Constructions Hydrauliques, Ed. A. Schleiss, Ecole Polyiechmique Fédérale de Lausame, Switzerland, ISSN1661-1179

[11] Heller P., Bollaert E. And Schleiss A. (2007) Comprehensive study of a multi-purpose run-of-river power plant with a holistic qualitative analysis. Jounal of River Basin Management. 8(3-4) 295-304

[12] Lamouroux N. AND SOUCHON Y. (2002) - - Simple predictions of in-stream habitat model outputs for fish habitat guilds in large streams. Freshwater Biology. 47 1531-1542

[13] Leach W. D. And Pelkey N. W. (2001) - Making Watershed Partnerships Work: A Review of the Empirical Literature. J. Woter Resour. Plng. Mgmt. 127(6) 378-385

[14] Lek S. ANd Guegan J.F. (1999) - Artificial Neural Networks as a tool in ecological modeling, an introduction. Ecol. Model $9039-52$
[15] LEYLAND G. (2002) - Multi-objective optimisation applied to industrial energy problems, these de doctorat $n^{\circ} 2572$, Ecole Polytechnique Fédérale de Lausame, Switzerland

[16] Meile T., Schleiss A. ANd Bolllat J.-L. (2005) Entwicklung des Abflussregimes der Rhone seit dem Beginn des 20. Jahrhunderts. Wasser: Energie. Luft - eati, énergie, air. $97(5 / 6) 133-142$

[17] OBACA M., WAGNer R. AND AL. (2001) - Modelling population dynamics of aquatic insects with artificial neural networks. Ecological Modelling. $146207-217$

[18] PelLaud M. (2007) - Ecological response of a milti-pumpose river development project using macro-invertebrates richness and fish habitat value, these de doctorat $n^{\circ} 3807$, Ecole Polytechnique Féderale de Lausanne, Switzertand

[19] Poff N.-L. AND ALLAN J. D. (1997) - The Natural Flow Regime: A paradigm for river conservation and restoration. Bioscience. 47(11) 769-784

[20] SILVERT W. (2000) - Fuzzy indices of environmental conditions. Ecological Modeling. $130111-119$

[21] SCHLEISS A. (2006) - Mögliche Synergien zwischen Hochwasserschutz, Flussrevitalisierung und Wasserkraft dank innovativer Mehrzweckprojekte. Wasser, Energie, Luft-eau. énergie, air. 98(1) 3-9

[22] Troisieme Correction Du Rhone (2006) - Plan sectoriel $3^{*}$ Correction du Rhône, Etat du Valais, Département des transports, de l'équipement et de l'environnement, Service des Routes et Cours d'Eau, Projet Rhone, Valais, Switzerland

[23] VALENTIN S. (1995) - Variabilité artificielle des conditions d'habitat et conséquences sur les peuplements aquatiques. effets écologiques des éclusées hydroélectriques en rivière, Laboratoire Hydroécologie Quantitative - Division Biologie des Ecosystèmes Aquatiques - CEMAGREF. Lyon, Université Claude Bernard-Lyon I. pp. 263

[24] Zhang L., Gove J.H. AND AL. (2005) - Spatial residual analysis of six modeling techniques. Ecological Modelling. 86 $154-177$ 